\section{Sarkopenie: Bedeutung und Definition im Wandel}

\author{
K. Norman ${ }^{1}$ \\ ${ }^{1}$ Leiterin der AG Ernährung und Körperzusammensetzung i. R.d. \\ Forschungsgruppe Geriatrie, Interdisziplinäres Stoffwechsel- \\ Centrum, Lipidambulanz, Lipidapherese, Ernährungsmedizin \\ und Sprechstunde für Altersmedizin, Charité - Universitäts- \\ medizin Berlin
}

Unter Sarkopenie (aus dem Griechischen: sarx = Fleisch; -penia = Mangel) versteht man die meist altersassoziierte Abnahme der Skelettmuskulatur. Die Ursachen für die Sarkopenie im Alter sind noch nicht eindeutig geklärt, scheinen aber multifaktoriell zu sein. Sie umfassen unter anderem endokrine und neuromuskuläre Veränderungen, eine genetische Prädisposition, aber auch Lifestyle-Faktoren wie geringe physische Aktivität und eine unzureichende Ernährung bzw. die im Alter häufig vorkommende Malnutrition. Die Folgen der Sarkopenie sind weitreichend und manifestieren sich unter anderem in einer reduzierten Mobilität mit erhöhter Sturz- und Frakturgefahr; und ebenfalls in metabolischen Beeinträchtigungen wie einer reduzierten Insulinsensitivität, die häufig bei der Sarkopenie auftritt. Insgesamt ist die Sarkopenie mit längeren Rekonvaleszenzzeiten, einer erhöhten Morbidität und Mortalität sowie einer verminderten Lebensqualität assoziiert und trägt auch zu einer höheren ökonomischen Belastung im Gesundheitswesen bei.

Da die Diagnose im klinischen Alltag noch selten gestellt wird, ist die Häufigkeit der Sarkopenie schwer einzuschätzen. Studien zufolge liegt die Prävalenz bei älteren Menschen zwischen 15 und 70 Prozent. Die unterschiedlichen Prävalenzen sind hauptsächlich auf Unterschiede bei der untersuchten Studienpopulation (Alter, klinische Situation), den Diagnosekriterien und der verwendeten Methodik zurückzuführen. Für die Erfassung der Muskelmasse im klinischen Bereich wird häufig die Dual-Röntgen-Absorptiometrie (DEXA) als Goldstandard angeführt, obwohl diese häufig im klinischen Alltag nicht verfügbar ist. Andere Methoden umfassen die wesentlich einfachere Bioelektrische Impedanzanalyse, die jedoch für die Messung der Skelettmuskulatur nicht ausreichend validiert ist, sowie aufwändigere Hilfsmittel wie die Computertomografie und Magnetresonanztomografie.

Um die Diagnosekriterien zu vereinheitlichen und die Diagnosestellung zu vereinfachen, wurden in letzter Zeit zwei Definitionen von internationalen Expertengremien wie der European Working Group on Sarcopenia in Older People (EWGSOP) oder der International Working Group on Sarcopenia (IWGS) vorgeschlagen. In beiden Definitionen werden eine erniedrigte Muskelmasse und eine Verringerung der
Mobilität oder Kraft für die Diagnosestellung verwendet. Während die Mobilitäts- und Kraftparameter zwischen den zwei Definitionen leicht unterschiedlich sind, wird durchgehend eine Abweichung von 2 Standardabweichungen der Muskelmasse bezogen auf die Körpergröße (Skelettmuskelmassenindex, $\mathrm{kg} / \mathrm{m}^{2}$ ) unterhalb des Mittelwertes einer gesunden jungen Referenzgruppe gleichen Geschlechts als klinischer Grenzwert für eine Verringerung der Skelettmuskulatur verwendet.

Der Bezug der Muskelmasse auf das Quadrat der Körpergröße ist jedoch nicht unproblematisch, da hierbei das Gesamtgewicht und die Körperzusammensetzung außer Acht gelassen werden. Bei Personen mit einem Übergewicht bzw. einer Adipositas wird demnach verhältnismäßig selten eine Sarkopenie durch den Skelettmuskelmassenindex (SMI, $\mathrm{kg} / \mathrm{m}^{2}$ ) festgestellt, da mit dem steigenden Körpergewicht auch die magere Körpermasse und die Muskelmasse steigen. Gerade das schiefe Verhältnis zwischen dem bei Senioren häufig höherem Körpergewicht und einer geringen Muskelmasse gilt jedoch als prognostisch ungünstig und geht mit einer eingeschränkten Leistungsfähigkeit und einer höheren Insulinresistenz bei älteren Menschen einher. Daher wurde auch von der Foundation for the National Institutes of Health (FNIH) Sarcopenia Project kürzlich der Quotient der Muskelmasse zum BMI als Diagnosekriterium für die Sarkopenie vorgeschlagen. Dadurch wird die Muskelmasse im Verhältnis zur gesamten Körpermasse betrachtet, und die Erfassung einer verringerten Muskelmasse auch bei höherem Gewicht wird möglich. In ersten Studien wurde das neue Diagnosekriterium im Vergleich zum SMI auch bereits validiert, indem ein engerer Bezug zur verringerten Mobilität und Leistungsfähigkeit nachgewiesen wurde.

Interessenkonflikt: Keine Interessenkonflikte.

\section{Bibliografie}

DOI http://dx.doi.org/10.1055/s-0035-1558067

Drug Res 2015; 65, Suppl. 1: S19-S19

(c) Georg Thieme Verlag KG Stuttgart · New York .

ISSN 2194-9379

\section{Korrespondenzadresse \\ PD Dr. Kristina Norman}

Leiterin der AG Ernährung und

Körperzusammensetzung

i. R.d. Forschungsgruppe Geriatrie

Charité - Universitätsmedizin Berlin

Reinickendorfer Straße 61

13347 Berlin

Interdisziplinäres Stoffwechsel-Centrum, Lipidambulanz, Lipidapherese, Ernährungsmedizin und Sprechstunde für Altersmedizin Charité - Universitätsmedizin Berlin

Augustenburger Platz 1

13353 Berlin

kristina.norman@charite.de 